\title{
Cemento-Ossifying Fibroma: Clinical, Radiological, and Histopathological Findings
}

\author{
Melda Pelin Akkitap ${ }^{1}$ (D) Birsay Gumru ${ }^{1}$ (iD) Ender Idman ${ }^{1}$ (D), Necip Fazil Erdem ${ }^{2}$ (D) , Zeynep Gumuser ${ }^{2}$ (D) Fatma Nihan Aksakalli $^{3}$ (iD \\ ${ }^{1}$ Marmara University, Faculty of Dentistry, Department of Oral and Maxillofacial Radiology, Istanbul, Turkey. \\ ${ }^{2}$ Marmara University, Faculty of Dentistry, Department of Oral and Maxillofacial Surgery, Istanbul, Turkey. \\ ${ }^{3}$ Istanbul University, Institute of Oncology, Department of Tumour Pathology and Oncological Cytology, Istanbul, Turkey.
}

Correspondence Author: Birsay Gumru

E-mail: bgumru@marmara.edu.tr

Received: $03.01 .2020 \quad$ Accepted: 15.10 .2020

\begin{abstract}
Cemento-ossifying fibroma, which is considered a benign mesenchymal odontogenic tumour of the jaws, is a type of fibro-osseous lesion characterized by slow growth and proliferation of fibrous cellular stroma containing osteoid, bone or cementum-like tissue. The aim of this study was to report a case of cemento-ossifying fibroma in the mandible with clinical, radiological, and histopathological findings and surgical treatment. A 37-year-old woman was admitted to our clinic with the complaint of gradually growing painless swelling in the right mandibular premolar area. Intra-oral examination revealed a bony hard and non-tender mass with intact overlying mucosa on the buccal and lingual aspects of the mandibular right premolar teeth. Periapical, panoramic, and cone beam computed tomography images revealed the presence of a multilocular hypodense lesion with bicortical destruction and expansion in the relevant area. Excisional biopsy of the lesion was performed and histopathological examination exhibited the definite diagnosis of cemento-ossifying fibroma. Clinical, radiological, and histopathological examination should be considered in the diagnosis and treatment planning of cemento-ossifying fibroma.
\end{abstract}

Keywords: Cemento-ossifying fibroma, fibro-osseous lesion, cone beam computed tomography

\section{INTRODUCTION}

Cemento-ossifying fibroma, derived from the periodontal ligament mesenchymal blast cells and capable of forming fibrous cellular tissue, osteoid, bone, cementum-like tissue, or a combination of such elements, is a benign mesenchymal odontogenic tumour of the jaws $(1,2)$.

Cemento-ossifying fibroma has been a puzzling and unclear term for many years (3). In 1971, the cemento-ossifying fibroma was first classified by the World Health Organization (WHO) under lesions containing cementum, including fibrous dysplasia, ossifying fibroma, and cementifying fibroma (4). According to the second classification of WHO in 1992, benign fibro-osseous lesions in the oral and maxillofacial region were grouped as osteogenic neoplasms and nonneoplastic bone lesions, separate cementifying fibroma and ossifying fibroma lesions were gathered under a single entity of "cemento-ossifying fibroma" because they showed different histological variants of the same type of lesion and belonged to the former category (5). Based on the WHO classification in 2005, the term "cemento-ossifying fibroma" was shortened to ossifying fibroma due to the fact that cementum-like material of odontogenic origin was found unexpectedly in fibromas located in the extragnatic regions, and the fact that cementum and bone were actually the same tissue that could be differentiated only by their relationship to tooth roots $(3,6)$. In addition, three ossifying fibroma forms (classical, psammomatoid juvenile, and trabecular juvenile) were distinguished $(3,7)$.

Recently, in 2017, the term "cemento-ossifying fibroma" was deemed to appropriately describe a benign mesenchymal odontogenic tumour specific to the tooth-bearing regions of the jaws (7). Cemento-ossifying fibromas can be classified as the central type arising from the periodontal ligament adjacent to the root apex and the peripheral type occurring exclusively in the soft tissues of the tooth-bearing regions (8).

The aetiology of cemento-ossifying fibroma remains obscure, but odontogenic, developmental, and traumatic factors have been proposed as predisposing factors due to stimulation of the periodontal ligament to produce and accumulate cementum and osteoid material $(1,8,9)$. 
The aim of this study is to report a case of cemento-ossifying fibroma in the mandible accompanied by clinical, radiological, and histopathological findings and surgical treatment.

\section{CASE PRESENTATION}

A 37-year-old woman, complaining of progressive painless swelling in the right mandibular premolar region for 1 year, was admitted to the Department of Oral and Maxillofacial Radiology, Faculty of Dentistry, Marmara University, Istanbul, Turkey. Her medical and dental histories were noncontributory.

Extra-oral examination revealed no facial asymmetry. There was no erythema, elevated local temperature, or palpable regional lymph nodes showing no evidence of inflammation.

In intra-oral examination, a bony hard and non-tender mass with intact overlying mucosa on buccal and lingual aspects of mandibular right premolars was observed (Figure 1a and b). The associated teeth were non-vital and mandibular right second premolar was mobile.

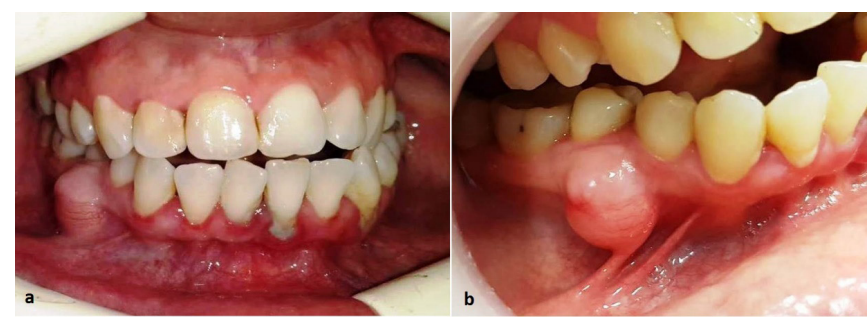

Figure 1a, b. Pre-operative intra-oral clinical view of the lesion.

The periapical radiograph and orthopantomogram (OPTG) demonstrated a multilocular radiolucent mass with welldefined borders and a sclerotic rim in the interradicular region of the mandibular right first and second premolars, and displacement of the second premolar. The coronal, sagittal, and axial plane cone beam computed tomography (CBCT) images revealed the presence of a well-defined hypodense lesion of approximately $9 \times 14 \times 8 \mathrm{~mm}$ with bicortical destruction and expansion without any cortical perforation in the area of interest. In addition, the continuity of the mandibular nerve within the lesion was disrupted (Figure 2a, b, and c).

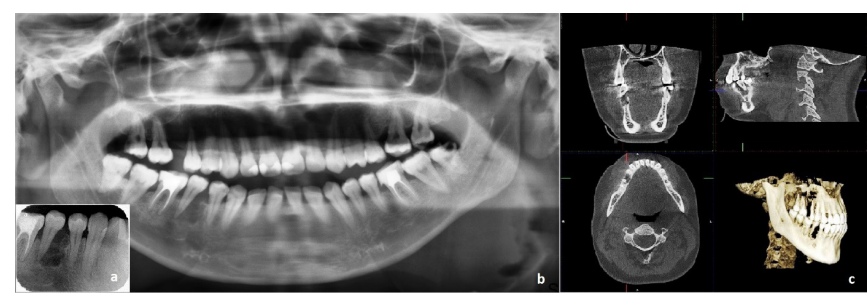

Figure $2 a, b, c$. Pre-operative periapical, OPTG, and CBCT (coronal, sagittal, axial planes, and $3 D$ reconstruction) images of the lesion.
Excisional biopsy of the lesion in conjunction with extraction of the mandibular right second premolar was performed under local anaesthesia. For the surgical exposure of the lesion, a crestal incision was performed to raise a fullthickness mucoperiosteal flap. Ostectomy was performed at clear margins of the bone with round and fissure burs under saline irrigation. Care was taken to protect the inferior alveolar nerve. Using proper osteotomes the lesion was excised totally as one piece. The lesion, which was macroscopically a lobular, elastic, and solid fibrotic mass attached to the root of mandibular right second premolar, could easily be separated from the periosteum. Since the lesion did not cause perforation in either the buccal or the lingual cortical bone plates and the periosteum was preserved, no graft material was used and the defect was left to heal like a routine extraction socket. The flap was sutured and primary closure was obtained. $500 \mathrm{mg}$ penicillin, $275 \mathrm{mg}$ naproxen sodium, and $0.1 \%$ chlorhexidine rinse two times a day were prescribed and continued for a week (Figure 3a, b, and c).

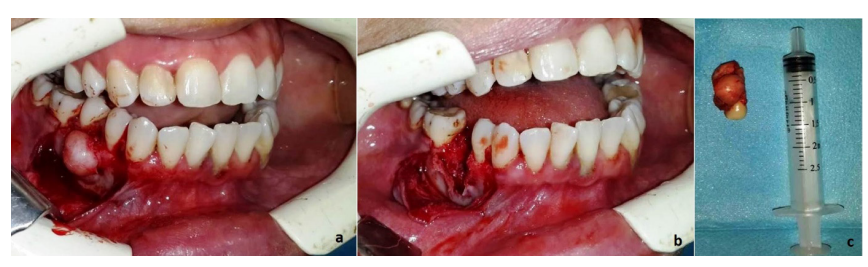

Figure $3 a, b, c$. Incision and exposure of the lesion during surgical procedure, and the excised mass.

In the microscopic examination, hematoxylin and eosin (H\&E) stained sections demonstrated well-demarcated, cellular fibroblastic stroma containing changeable amount of mineralized areas, osteoid, immature bone, and spherical cementum-like tissues confirming the diagnosis of cementoossifying fibroma (Figure $4 a$ and $b$ ).

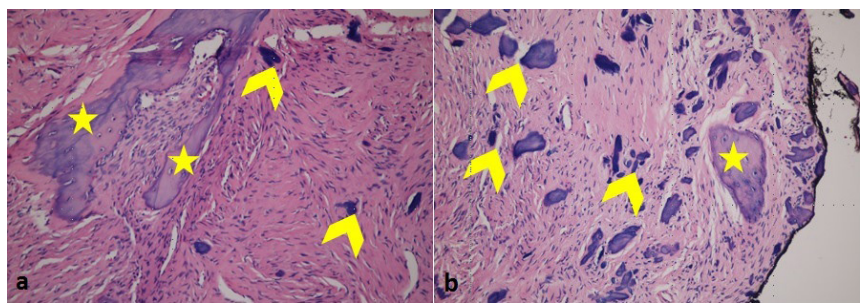

Figure $4 a, b$. Mineralized areas, immature bone trabeculae $\left({ }^{*}\right)$, and small, spherical cementum-like structures ( Л) (H\&Ex200).

Post-operatively, the area recovered uneventfully. No recurrence was detected clinically and radiologically at the 1-year follow-up (Figure 5a and b). 


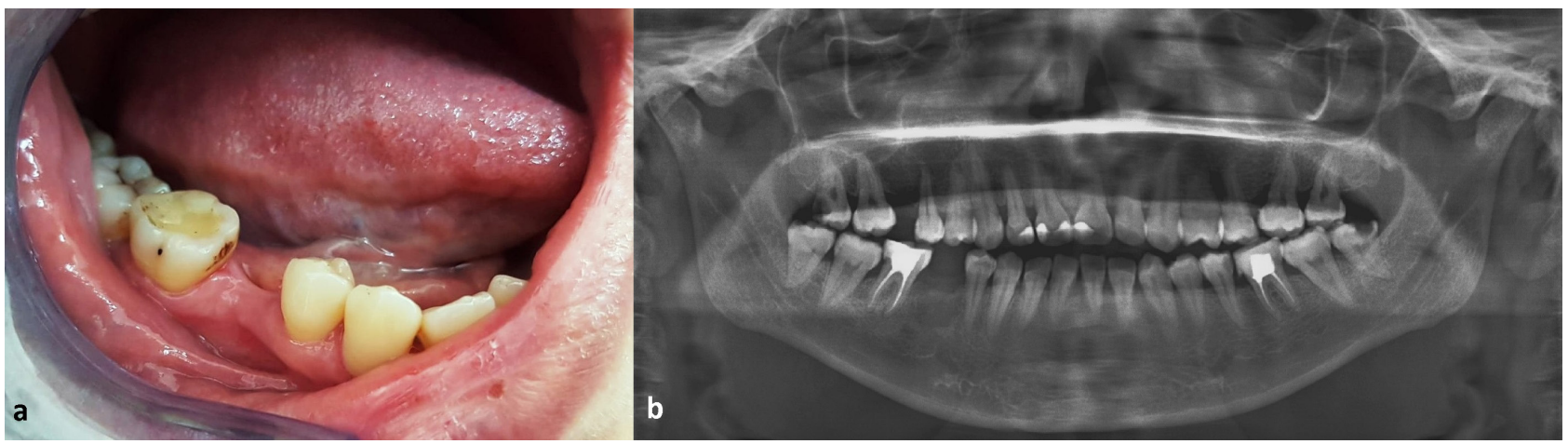

Figure 5a, b. Post-operative 1-year follow-up intra-oral clinical view and OPTG image.

\section{DISCUSSION}

Cemento-ossifying fibroma is a rare benign odontogenic neoplasm characterized by replacement of normal bone with fibrous tissue $(2,10,11)$. Although it has been reported to occur in children, adolescents, and elderly adults, it is most commonly diagnosed in the second to fourth decades of life $(12,13)$. Women are affected more often than men with a female-to-male ratio of up to $5: 1(2,10,14,15)$.

Cemento-ossifying fibroma has a predilection for the premolar-molar region of the mandible and is usually small in size (16). However, the maxilla and paranasal sinuses may be involved and the lesion may gradually become large in these areas as there is more space for expansion $(12,17)$.

Clinically, cemento-ossifying fibroma usually presents as a slow-growing and asymptomatic intra-bony mass but it may cause facial asymmetry or bone fracture when it becomes large in size $(1,2,11)$. Although teeth in proximity with the lesion usually preserve their vitality, pain or paraesthesia may occur if pressure is exerted on a neighbouring nerve (11). It is mostly solitary, but rarely may present as multiple synchronous lesions which are isolated or a component of hyperparathyroidism-jaw tumour syndrome $(7,18)$.

The radiological features of cemento-ossifying fibroma reveal a well-defined mostly unilocular or in minority of cases multilocular radiolucency with or without radiopaque foci associated with the degree of calcification $(1,18)$. In the initial phase, cemento-ossifying fibroma manifests as a radiolucent lesion with the absence of internal radiopacity. With the increasing tumour maturity, radiopaque masses which may coalesce to form a large radiopaque focus surrounded by radiolucent border emerge (9). There are three distinct patterns of radiographic margins, which are defined lesion without sclerotic border (40\%), defined lesion with sclerotic border (45\%), and lesion with ill-defined border implying a fast growing tumour $(15 \%)(11,19)$. A particular diagnostic radiographic hallmark is the centrifugal growth pattern instead of a linear one, and therefore the lesions grow uniformly in all directions and present as a round tumour mass $(9,11,19)$. Cemento-ossifying fibroma may lead to root resorption, divergence or mobility of the adjacent tooth $(9,19)$. Additionally, buccal and lingual bone expansion unaccompanied by cortical perforation may be detected $(1,11,12)$.

Fibrous dysplasia, cemento-osseous dysplasia, giant cell granuloma, calcifying odontogenic cyst (Gorlin cyst), calcifying epithelial odontogenic tumour (Pindborg tumour), adenomatoid odontogenic tumour, and osteogenic sarcoma should be regarded in radiological differential diagnosis. Fibrous dysplasia has a characteristic "ground glass" appearance and blending margin with the surrounding bone. However, cemento-ossifying fibroma is consistently well-defined and discriminated from the circumscribing bone. Cemento-osseous dysplasia demonstrates broad sclerotic borders and is multifocal opposed to cementoossifying fibroma. Giant cell granuloma, which is more common in the mandible affecting the earlier age groups, shows a slightly granular pattern of fine calcification with poorly-defined internal septa and causes displacement or resorption of tooth roots. Gorlin cysts, Pindborg tumours, and adenomatoid odontogenic tumours are frequently involved with impacted teeth. On condition that they are not associated with impacted teeth, the definitive diagnosis is based on histological appearance. The well-defined border of the cemento-ossifying fibroma facilitates the differentiation from osteogenic sarcoma which presents cortical bone destruction and invasion into the surrounding soft tissues and periodontal ligament space $(8,9,13,19,20)$.

Histologically, cemento-ossifying fibroma is composed of well-vascularized fibrocellular tissue capable of forming immature bone trabeculae and cementoid $(10,12,14,15)$. The bone trabeculae are variable in size and often show a combination of woven and lamellar pattern. Bone usually demonstrates peripheral osteoid and osteoblastic rimming. The cementoids are basophilic spherical bodies representing peripheral brush border that blend into adjacent connective tissue and also have a smooth contour with cells $(16,20,21)$. Nevertheless, cementum and immature bone trabeculae may also be seen in other fibro-osseous lesions such as fibrous dysplasia and cemento-osseous dysplasia, so this appearance is not specific for cemento-ossifying fibroma $(10,14,22)$. On the grounds of this, a correlation of clinical, radiological, and histopathological findings is required for a definite diagnosis. 
Enucleation with curettage is the treatment of choice for small lesions, and surgical resection and reconstructive surgery are indicated for larger lesions $(16,17,20,21)$. Enucleation is unchallenging because it is a clearly defined lesion that easily detaches from the surrounding bone. This is the most important clinical and per-operatory feature for differentiation from fibrous dysplasia. Radiotherapy has been confirmed to be ineffective and contraindicated due to its inductive effect for malignant transformation (12). The prognosis of cemento-ossifying fibroma is favourable and recurrence, reported as high as $12 \%$, is unusual $(13,15-$ $18,21)$. The recurrence rate of maxillary cemento-ossifying fibromas is higher in comparison to mandibular ones as a consequence of further complicated surgical removal and larger size at the time of diagnosis $(8,20)$.

Our case was a 37-year-old female patient who presented with an asymptomatic slow-growing mass in the mandibular premolar-molar region. Radiographic examination revealed a well-defined, multilocular lesion with a sclerotic border. The early stage lesion manifested as radiolucency without internal radiopacity. Furthermore, expansion and destruction of the buccal and lingual cortices were detected without any cortical perforation. Histopathological findings were similar to the characteristics of cemento-ossifying fibroma. Because the lesion was small in size, treatment was performed by enucleation and no recurrence was observed in the 1-year follow-up.

\section{CONCLUSION}

Clinical, radiological, and histopathological examination should be implemented in the diagnosis and treatment planning of cemento-ossifying fibroma. Asymptomatic benign fibro-osseous lesions may lead to problems such as complicated surgical procedures, post-operative complaints, or prosthetic needs as a result of extraction of teeth associated with the lesion. In conclusion, patients should be instructed not only about the importance of early detection of the lesion, but also the necessity of routine periodic checkups for avoidance of recurrence or future complications after treatment.

\section{ACKNOWLEDGEMENT}

This study is presented as a poster at the $25^{\text {th }}$ International Dental Congress of Turkish Dental Association, Istanbul, Turkey, September 4-7, 2019.

\section{REFERENCES}

[1] Liu Y, Wang H, You M, Yang Z, Miao J, Shimizutani K, Koseki T. Ossifying fibromas of the jaw bone: 20 cases. Dentomaxillofac Radiol. 2010;39:57-63.

[2] Pandey V, Sharma A, Sudarshan V. Cemento-ossifying fibroma - a rare case report with review of literature. IJCMR 2016;3:2681-2682

[3] Speight PM, Takata T. New tumour entities in the 4th edition of the World Health Organization Classification of Head and
Neck tumours: odontogenic and maxillofacial bone tumours. Virchows Arch 2018;472:331-339.

[4] Pindborg JJ, Kramer IRH. Histological typing of odontogenic tumours, jaw cysts and allied lesions. International histological classification of tumours. Geneva: WHO;1971. p.31-34.

[5] Kramer IR, Pindborg JJ, Shear M. The World Health Organization histological typing of odontogenic tumours; introducing the second edition. Eur J Cancer B Oral Oncol 1993;29:169-171.

[6] Reichart PA, Philipsen HP, Sciubba JJ. The new classification of Head and Neck Tumours (WHO) - any changes? Oral Oncol 2006;42:757-758.

[7] El-Mofty SK, Nelson B, Toyosawa S. Ossifying fibroma. ElNaggar AK, Chan JKC, Grandis JR, Takata T, Slootweg PJ, editors. WHO Classification of Head and Neck Tumours. 4th ed. Lyon: IARC Press; 2017. p. 251-252.

[8] Bala TK, Soni S, Dayal P, Ghosh I. Cemento-ossifying fibroma of the mandible a clinicopathological report. Saudi Med J 2017;38:541-545.

[9] Mithra R, Baskaran P, Sathyakumar M. Imaging in the diagnosis of cemento-ossifying fibroma: A case series. J Clin Imaging Sci 2012;2:52.

[10] Sridevi U, Jain A, Turagam N, Prasad MD. Cemento-ossifying fibroma: A case report. Adv Cancer Prev 2016;1:111.

[11] Rani A, Kalra N, Poswal R, Sharma S. Cemento-ossifying fibroma: Report of a case and emphasis on its diagnosis. Indian J Multidiscip Dent 2017;7:140-143.

[12] Dalghous A, Alkhabuli JO. Cemento-ossifying fibroma occurring in an elderly patient: A case report and a review of literature. Libyan J Med 2007;2:95-98.

[13] Wanzeler AMV, Rohden D, Arús NA, Silveira HLD, Hildebrand LC. Central cemento-ossifying fibroma: clinical-imaging and histopathological diagnosis. Int J Odontostomat 2018;12:233-236.

[14] Silvestre-Rangil J, Silvestre FJ, Requeni-Bernal J. Cementoossifying fibroma of the mandible: Presentation of a case and review of the literature. J Clin Exp Dent 2011;3:66-69.

[15] Peravali RK, Bhat HH, Reddy S. Maxillo-Mandibular Cementoossifying fibroma: A rare case report. J Maxillofac Oral Surg 2015;14:300-307.

[16] Kharsan V, Madan RS, Rathod P, Balani A, Tiwari S, Sharma S. Large ossifying fibroma of jaw bone: a rare case report. Pan Afr Med J 2018;30:306.

[17] Reddy R, Sarkar P, Manuel RA, Saxena D, Hoisala VR. Cementoossifying fibroma: A case report. IJSS Case Reports and Reviews 2016;3:13-15.

[18] Neville BW, Damm DD, Allen CMA, Chi AC. Oral and Maxillofacial Pathology. 4th ed. St. Louis, Missouri: Elsevier; 2016.

[19] Swami A, Kale L, Mishra S, Choudhary S. Central ossifying fibroma of mandible: A case report and review of literature. J Indian Acad Oral Med Radiol 2015;27:131-135.

[20] Atul SK, Bishen KA, Shitalkumar GS. Understanding massive maxillofacial central cement-ossifying fibroma. Indian J Stomatol 2014;5:148-150.

[21] Koury ME, Regezi JA, Perrott DH, Kaban LB. Atypical fibroosseous lesions: diagnostic challenges and treatment concepts. Int J Oral Maxillofac Surg 1995;24:162-169.

[22] Regezi JA, Sciubba JJ, Jordan RCK. Oral Pathology: Clinical Pathologic Correlations. 7th ed. St. Louis, Missoouri: Elsevier; 2016. 
